\title{
Integrated Magnetic Array for Bio-object Sensing and Manipulation
}

\author{
Faisal T. Abu-Nimeh and Fathi M. Salem \\ Circuits, Systems, and Neural Networks Lab \\ Dept. of Electrical \& Computer Engineering \\ Michigan State University, East Lansing, USA \\ \{asd1815, salem\}@egr.msu.edu
}

\begin{abstract}
Magnetic molecular-level interrogation, manipulation, and diagnosis are emerging as lab-on-chip platforms. These platforms entail low-cost, low-power, portable, and high efficiency integrated implementations. We introduce an all-integrated programmable 16x16 magnetic coil array chip for sensing and actuating small single bio-objects or collaboratively manipulating larger ones. The die-size is $1.5 \times 1.5 \mathrm{~mm}^{2}$ designed in bulk $0.5 \mu \mathrm{m}$ CMOS technology. The integrated design does not require any external magnetic source. It relies on the Hall effect generated by the smallest permissible vertical coil inductors (in this reported technology, the smallest inductor's planar area is $6 \mu \mathrm{mx6} \mu \mathrm{m})$. The coil array is selectively and dynamically controlled. Each cell, composed of the coil and its logical control circuitry, can detect small objects in the order of $1 \mu \mathrm{m}$ diameter as well as emit eight programmable magnetic field levels for manipulation. All array sensing and driving components are shared to reduce the overall imprint. Also, they are tuned to work at $900 \mathrm{MHz}$ incorporating high-speed serial row/column switching for seamless pseudoparallel operation.
\end{abstract}

Index Terms-On-Chip Magnetic Force, Collaborative Object Manipulation, Bio-sensor, Lab-on-Chip.

\section{INTRODUCTION}

There exist several technologies besides CMOS with better performance and sensitivity, e.g. BJT and MEMs. Bulk CMOS, however, provides several feature advantages over these technologies that include being inexpensive, scalable, low power, reliable, and easily integrated with the computational power of digital electronics. There is a recent trend in the bio-sciences to develop lab-on-chip platforms to detect, interrogate, or manipulate bio-objects for infection diagnosis and treatment [1]. The trend is also towards replacing optical fluorescence-based tag sensors with their magnetic-based counter parts for various advantages including compactness, device portability, and high sensitivity and selectivity.

Existing magnetic bio-sensors and particle manipulators often require an external source to generate the reference magnetic field as in [2], [3] or they perform manipulation on a single object as in [1]. Additionally, other researchers use the CMOS Hall effect to sense and detect magnetic beads using "static" arrays and chambers [4]. In contrast, in this work, we introduce a programmable 16x16 compact array for dynamic object manipulation and sensing. All components of the design are integrated on the same die without the need for specialized post processing, magnetized materials, or packaging. Present state-of-the-art designs are limited to sensing only [5], actuation (manipulation) only [6], or static designs which require user intervention or special microfluidic packaging. Our platform sets the stage for integrated capabilities to manipulate and sense multiple small objects in pseudo-parallel operations or larger single objects with a collaborative execution scheme. Each cell in the array can generate 8 programmable magnetic field levels and can sense magnetic beads with a diameter of at least $1 \mu \mathrm{m}$; thus, giving the user more functionality and flexibility in a unified standard package.

The proposed operational concepts of the coil array can be explained as follows. Using a technique analogous to switching regulators (switched-mode power supplies), the average output current of the amplifier can be controlled by switching the oscillator on/off at select durations. The durations are specific intervals that contain different fractions of the oscillation periods to provide different DC components. Thus, the DC magnetic field, and correspondingly the DC force, can be selectively commanded to generate forward (positive) or backward (negative) actuation on magnetized objects. In one targeted application, namely actuating a magnetic bead in fluid, it is anticipated that the high frequency components would be filtered out by the fluidic medium and primarily low and DC components would contribute to the actuation of the bead. Thus non-contact sensing and actuation of magnetized beads is easily achieved with the proposed integrated platform. The actual strategies of switching operations for best (sensing and/or actuation) performance is relegated to software in order to gain flexibility for broad choices of the sensing and control space. In the following sections, we proceed with a bottom-up description of the bio-chip, first the building blocks, then the overall system.

\section{BUILDING BLOCKS}

\section{A. Power Amplifier}

The class E power amplifier (PAE) [7] provides an efficient design of the driving engine for the array. In order to increase the amplifier's overall power output and to digitally control it, one replaces the single NMOS switch with a parallel NMOS configuration [8]. Figure 1 shows the parallel NMOS switches (we use minimum size W/L). The eight inputs are connected to a 3-to-8 thermometer decoder to reduce the number of I/O pins required to control the PAE, and to facilitate binary-word 


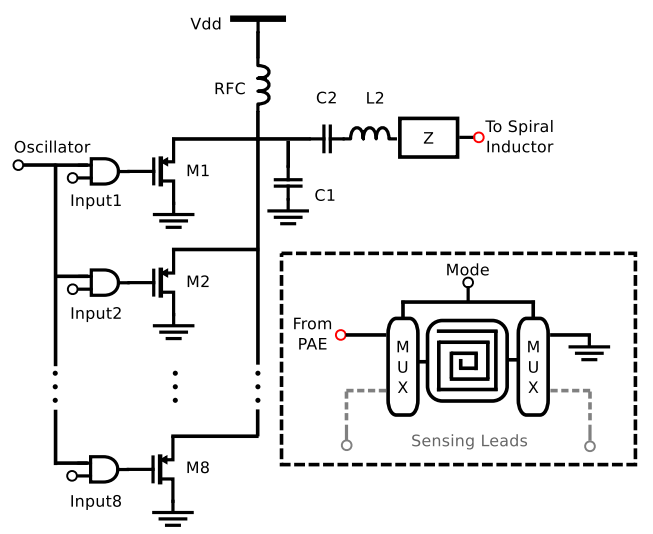

Fig. 1. A variable gain class E power amplifier tuned at $900 \mathrm{MHz}$. The dashed box is a simplified version showing how the same coil can be shared for sensing and actuation. In actuation mode, the PAE is connected to a single coil, the other end of the coil is connected to ground

increase/decrease in magnetic field actuation [9]. In figure 1, RFC represents a large on-chip inductor that provides a DC path from the supply and it is approximated as an open circuit at RF. The inductance L2 is designed to be $30 \mathrm{nH}$ and $\mathrm{C} 2$ is determined such that the PAE is tuned to $900 \mathrm{MHz}$. Using the lowest output power, the PAE generates $150 \mu \mathrm{A}$ (peak-to-peak) when driving a single coil actuator.

\section{B. PAE Oscillator}

A simple ring oscillator, tuned at $900 \mathrm{MHz}$, is chosen in this design to reduce the complexity of the system and minimize real-estate. Ring oscillators are known to fluctuate with temperature changes and drift with time, therefore, if necessary, one can replace it with a Phase-Locked-Loop. This platform is intended for temperature controlled bio-materials where the chip is assumed to operate around room temperature. The number of stages (gates) is chosen to be three, and the W/L sizes of each gate are adjusted to obtain an average delay of $0.10422 \mathrm{~ns}$ in the fabrication process. The output frequency is given as $f=\frac{1}{2 * N * T_{d}}$ where $f$ is the frequency, $N$ is the number of stages and $T_{d}$ is the (average) time delay for each stage. Accordingly, $f$ is calculated to be approximately $900 \mathrm{MHz}$.

The Class E Power Amplifier described in section II-A is controlled by a 3-to- 8 thermometer decoder, where the smallest possible input '000' translates to '00000001' output. This keeps the PAE turned on at all times unless the oscillator is switched off. Consequently, we have replaced the inverter in the first stage of the oscillator with an NAND gate to allow switching off the oscillator and consequently, the PAE. Figure 2 shows the average (DC component) of the periodic output of the PAE as a result of different switching intervals.

\section{Vertical Inductors}

The magnetic field generated in the array is based on the size of the inductor, the current passing through it, and the medium surrounding it. In this paper, the on-chip inductors were prototyped using all available 3 metal layers to constitute the

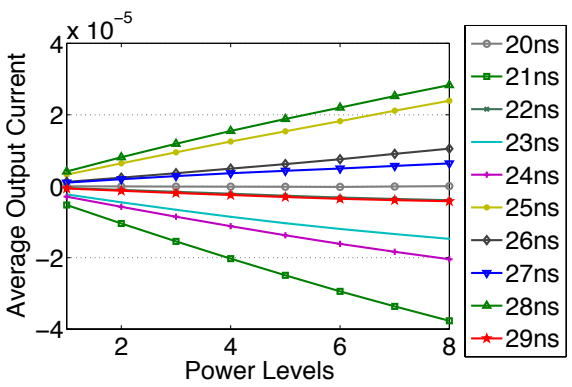

Fig. 2. The average output current for different switching periods (20ns to $29 \mathrm{~ns}$ ). The magnitude is maximum at $28 \mathrm{~ns}$ and $21 \mathrm{~ns}$ switching periods.

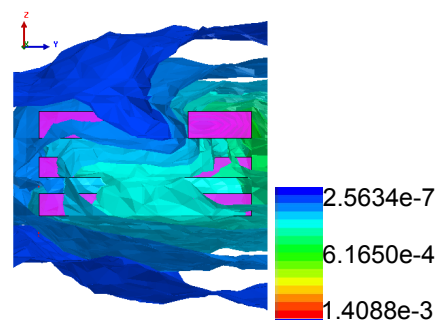

Fig. 3. [3D Side view] Simulated magnetic field shown in green/blue as a result of passing $1.5 \mathrm{~mA}$ through it ( 3 metal layers highlighted in pink). The B-field near the surface is approximately $0.4 \mathrm{mT}$

smallest possible size in OnSemi $0.5 \mu \mathrm{m}$ CMOS technology. Each coil measures $6 \times 6$ microns in the plane with a coil inductance of $14.42495 \mathrm{pH}$ and a resistance of $0.52 \mu \mathrm{Ohms}$. The 6x6 microns inductors were modeled (pre-fabrication) to assure enough magnetic field output per coil. The magnetic flux density generated is characterized (see figure 3) with the assistance of an EM simulator (Ansoft Designer 4) using the proprietary technology parameters to ensure precision. The simulation shows that using the largest possible PAE current produces a magnetic flux close to $0.4 \mathrm{mT}$.

Furthermore, a test circuit has been fabricated to quantify the ability of these tiny inductors to manipulate magnetized objects. The test circuit consists of a PAE, 3-to-8 thermometer decoder, and a mini-sized 6x6 micron coil. The passivation layer covering the coils has been removed to allow precise measurements. To measure the generated magnetic field of the fabricated coil, we have used an Atomic Force Microscopy (AFM) system with a magnetized tip (MAGT) fabricated by AppNano. The cantilever's tip height is around $15 \mu \mathrm{m}$ and its radius of curvature (ROC) is around $35 \mathrm{~nm}$. The cantilever's tip was placed on top of the "exposed" test chip surface as shown in figure 4 . The magnetic field of the coil has been detected by recording the measured deflection of the cantilever using the AFM optical laser sensor. The force exerted on the cantilever's tip is then computed. For each power level, we operated the AFM system to conduct 256 measurements which were then averaged. Therefore, a total of 2048 experimental runs were executed to measure 8 different (cantilever deflection) force levels. The experiment runs were separated by a 5 second timeout period to eliminate possible 


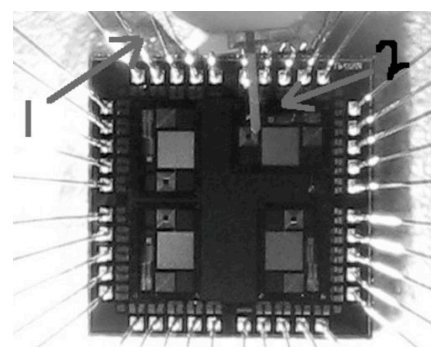

Fig. 4. (Top View) Magnetic Cantilever placed on top of the exposed coil cell (shown) to quantify its generated magnetic field. Number (1) points to the cantilever body and (2) points to its tip.

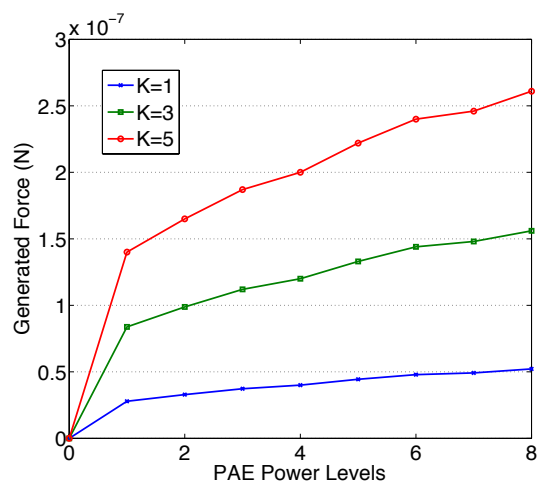

Fig. 5. The forces (in Newton) generated on the cantilever tip by a single onchip coil using different power levels. $K$ represents different spring constants provided by the cantilever's manufacturer.

correlation between different driving power levels. In figure 5, the middle curve depicts the nominal magnetic field-generated forces for the 8 driving power levels.

\section{Sensing Logic}

Using the output frequency of a cross coupled LC oscillator, as shown in figure 6 , it is possible to detect magnetized particles in the order of $1 \mu \mathrm{m}$. The method used in detection relies on the effective inductance of the coil: For normal operation, the oscillation resonates at frequency $f=1 /\left(2 \pi \sqrt{L C_{1}}\right)=$ $900 \mathrm{MHz}$. Nevertheless, when introducing a new magnetic medium, e.g a magnetic bead, on top of the coil, its effective inductance is going to increase- resulting in a decrease in the oscillating frequency. Then, a simple integrated frequency counter can detect the change in frequency.

The $6 \times 6$ microns vertical inductor of $14.42495 \mathrm{pH}$ would require a $2.19 \mathrm{nF}$ capacitor to oscillate at $900 \mathrm{MHz}$. Therefore, a LC tank is constructed with appropriate cross coupled MOS transistors for the oscillator. To verify the sensing abilities of the coil, we incorporated the Invitrogen MyOne Dynabead's model, using its physical specifications as well as the B$\mathrm{H}$ hysteresis curves provided by the manufacturer, into an EM simulator. Then, we simulated the vertical coil with the bead (suspended in distilled water) about $2 \mu \mathrm{m}$ away from the coil's surface. The EM simulator subsequently recomputed the inductance of the vertical coil to be $14.28024 \mathrm{pH}$. Several simulations have shown that the oscillation frequency

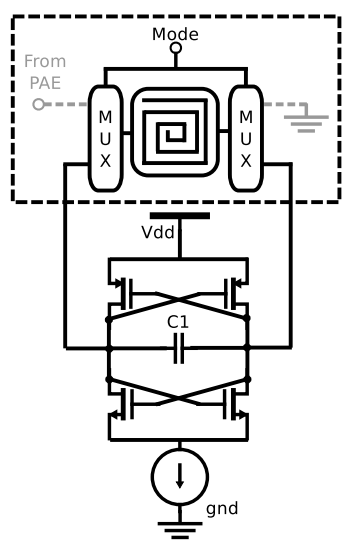

Fig. 6. Cross coupled LC oscillator. The output frequency of the oscillator is also tuned to $900 \mathrm{MHz}$. $C_{1}$ is $\sim 11 \mathrm{pF}$.

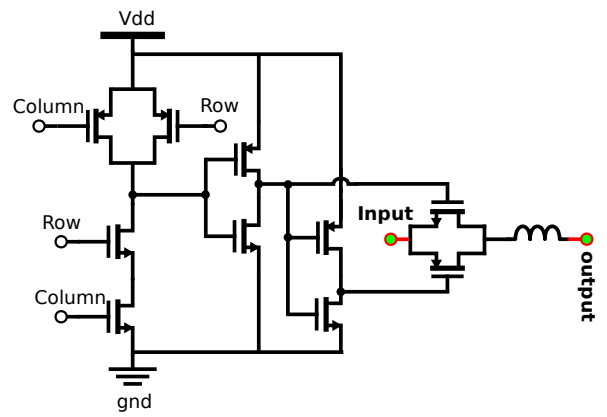

Fig. 7. A single cell with its control logic. The input of the cell is highlighted in red and is directly connected to the Sensing Oscillator or the PAE.

decreases below $900 \mathrm{MHz}$ (in shifts ranging from $0.5 \mathrm{MHz}$ to $5 \mathrm{MHz}$ ). Such shift ranges are easily detectable by the frequency counter.

\section{E. Logic Control}

Each cell in the 16x16 array needs to be accessed individually. Therefore, two 4-to-16 decoders are used to selectively link any inductor load to the driving circuits (the cross coupled LC oscillator and PAE). The decoders operate as in Random Access Memory (RAM) designs. Each input enables one of the sixteen rows and columns available. In order for the driving circuits to access a single coil cell, a high-speed control signal (switching at $40 \mathrm{MHz}$ ) is used to link a specific cell to the driving circuity. Figure 7 depicts the basic logic transmissiongate (switch) for each inductor cell. If cross-talk and wireloading is tolerated, a single NMOS switch can be used instead. However, due to the noise and coupling generated by neighboring cells, using NMOS switches exhibits negative influence on the performance of this array.

\section{ARChitecture And System InTEgRation}

Integrating all components together on the same die requires careful considerations in terms of routing, isolation, and coupling among neighboring coils. Moreover, careful considerations are due for proper placement of RF and digital circuits with noisy sources, and forming the array shape 


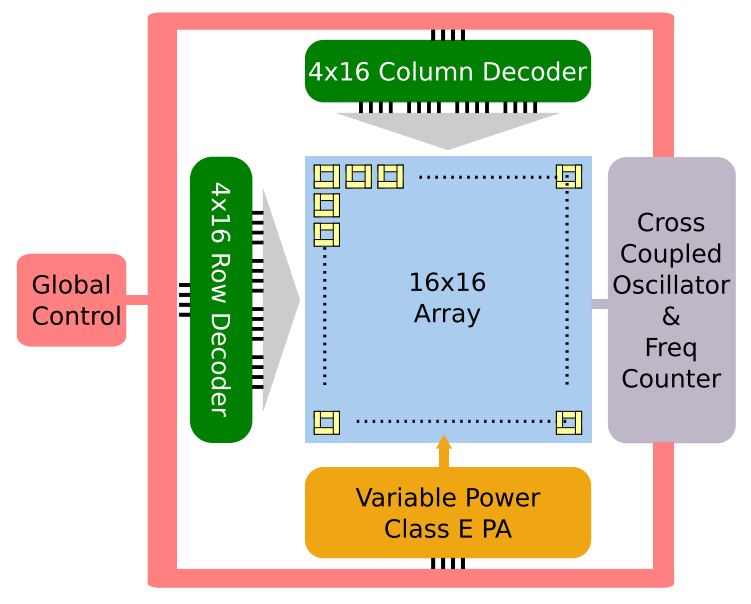

Fig. 8. System-level block diagram.

according to the targeted applications. The modular design should consider distributing heat equally across the entire array to maintain consistent interactions with the bio-objects of interest. The overall system, shown in figure 8, has 3degrees-of-freedom: the shape of the array, different magnetic field levels per cell, and the array control scheme e.g. spiral, pyramidical, etc. Accordingly, with different designs, one can create an arbitrarily collective 3D scenarios for manipulation. For example, it might be desired to generate a bowl-shaped magnetic field using a square array. To that end, one can program the digital controller to set the edges of the array on maximum power ' 111 ' and reduce the power linearly along the $\mathrm{X}$ and $\mathrm{Y}$ axes in a gradient fashion towards the center of the square. More details on these schemes are the subject of future reports.

A prototype array was fabricated in $0.5 \mu \mathrm{m}$ 3-Metal-2-Poly CMOS. We used the $1.5 \mathrm{mmx} 1.5 \mathrm{~mm}$ size to fill it up with $16 \times 16$ min-sized inductor cells. This prototype is an asymmetrical 16x16 array suitable for singular and collaborative object manipulation with an estimated power consumption of $67 \mathrm{~mW}$. The array filled out the entire upper portion of the die as shown in figure 9. The lower portion of the design, contains the PAE, frequency counter, digital control, and test circuits. The LC oscillator was not included in this run. The components have been intentionally separated and isolated to reduce coupling (cross-talk) and improve the performance of actuation. The control logic is applied directly to the chip from an external device using the I/O pins. Furthermore, the I/O pads on the upper half of the die have been removed to minimize the noise generated from the bonding wires and allow AFM testing without damaging or shorting the I/O pins. The digital circuity and switching overhead occupies $51.450 \mu \mathrm{mx} 34.5 \mu \mathrm{m}$. Finally, it is possible to modify these arrays and move all the digital components to the die sides by creating a wide bus for the interconnects. This permits the coil array to be closely stacked and compact. However, careful component isolation to minimize coupling and noise may be challenging. We plan to report on this third design in a future publication.

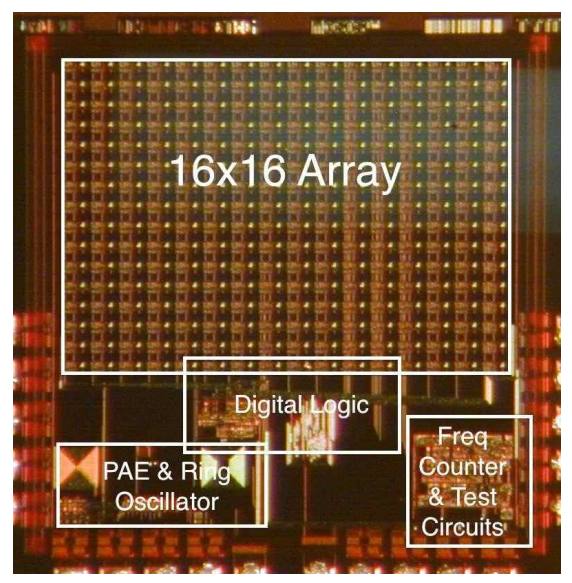

Fig. 9. Photograph of $1.5 \mathrm{~mm} \times 1.5 \mathrm{~mm}$ CMOS die.

\section{CONCLUSION}

We present an efficient integrated design to implement a lab-on-chip programmable magnetic field generator and object sensor using standard CMOS technology without the need for external magnetics or special fabrication and packaging processes. The introduced platform is suitable for single or collaborative object sensing and manipulation. The system architecture design has 3 degrees of freedom: variable magnetic field, programmable control scheme, different vertical inductor and array shapes and sizes. This makes it possible to dynamically create an unlimited number of magnetic force profiles and contours needed to interact and sense different bio-objects.

\section{REFERENCES}

[1] X. Janssen, L. van IJzendoorn, and M. Prins, "On-chip manipulation and detection of magnetic particles for functional biosensors," Biosensors and Bioelectronics, Jan 2008.

[2] S.-J. Han, H. Yu, B. Murmann, N. Pourmand, and S. Wang, "A highdensity magnetoresistive biosensor array with drift-compensation mechanism," Solid-State Circuits Conference, 2007. ISSCC 2007. Digest of Technical Papers. IEEE International, pp. 168 - 594, Feb 2007.

[3] Y. Liu, N. Sun, H. Lee, R. Weissleder, and D. Ham; "Cmos mini nuclear magnetic resonance system and its application for biomolecular sensing," Solid-State Circuits Conference, 2008. ISSCC 2008. Digest of Technical Papers. IEEE International, pp. 140 - 602, Feb 2008.

[4] E. P. Dupont, E. Labonne, C. Vandevyver, U. Lehmann, E. Charbon, and M. A. M. Gijs, "Monolithic silicon chip for immunofluorescence detection on single magnetic beads," Anal Chem, vol. 82, no. 1, pp. 49 52, Jan 2010.

[5] H. Wang, Y. Chen, A. Hassibi, A. Scherer, and A. Hajimiri, "A frequencyshift cmos magnetic biosensor array with single-bead sensitivity and no external magnet," Solid-State Circuits Conference - Digest of Technical Papers, 2009. ISSCC 2009. IEEE International, pp. 438 - 439, 2009.

[6] H. Lee, Y. Liu, D. Ham, and R. Westervelt, "Integrated cell manipulation system-cmos/microfluidic hybrid," Lab on a Chip, vol. 7, no. 3, pp. 331-337, 2007.

[7] F. Abu-Nimeh and F. Salem, "Digital class e power amplifier for the rf band," Mechatronics and Automation, Proceedings of the 2006 IEEE International Conference on, pp. 1548 - 1552, May 2006.

[8] P. Cruise, C.-M. Hung, R. Staszewski, O. Eliezer, S. Rezeq, K. Maggio, and D. Leipold, "A digital-to-rf-amplitude converter for gsm/gprs/edge in 90-nm digital cmos," Radio Frequency integrated Circuits (RFIC) Symposium, 2005. Digest of Papers. 2005 IEEE, pp. $21-24$, Jun 2005.

[9] F. Abu-Nimeh and F. Salem, "Cmos integrated electro-magnetic force actuator," Circuits and Systems, 2006. MWSCAS '06. 49th IEEE International Midwest Symposium on, vol. 2, pp. 395 - 398, Jul 2006. 